\title{
SUPORTE DE CABEÇA PARA CIRURGIA DA COLUNA VERTEBRAL NOTA TÉCNICA
}

\author{
HEAD SUPPORT FOR SPINAL SURGERY. TECHNICAL NOTE \\ SOPORTE DE CABEZA PARA CIRÚGIA DE COLUMNA VERTEBRAL. NOTA TÉCNICA
}

Guilherme Zanini Rocha ${ }^{1}$, Aluízio Augusto Arantes Junior² ${ }^{2}$ José Guilherme Mendes de Oliveira ${ }^{3}$, Fernando Flavio Vasconcelos Gonçalves ${ }^{4}$, Julio César Boynard Santiago ${ }^{5}$, Sebastiāo Silva Gusmão6

\begin{abstract}
RESUMO
Descreve-se um suporte de cabeça para cirurgia da coluna vertebral, que permite a colocação da cabeça do paciente em alinhamento neutro com o corpo e monitorização da posição de olhos, boca, nariz e tubo orotraqueal, evitando danos decorrentes de mau posicionamento durante a cirurgia.
\end{abstract}

Descritores: Coluna vertebral/Cirurgia; Anestesia; Segurança; Complicações; Posicionamento do paciente; Decúbito ventral.

\begin{abstract}
We describe a head support for spinal surgery that allows the placement of the patient's head in neutral alignment with the body and monitoring the position of the eyes, mouth, nose and orotracheal tube, preventing damages from poor positioning during surgery.
\end{abstract}

Keywords: Spine/Surgery; Anesthesia; Safety; Complications; Patient positioning; Prone position.

\section{RESUMEN}

Se describe un soporte de cabeza para la cirugía de columna vertebral que permite la colocación de la cabeza del paciente en alineación neutra con el cuerpo y el control de la posición de ojos, boca, nariz y tubo oro-traqueal, evitando los daños causados por el posicionamiento incorrecto durante la cirugía.

Descriptores: Columna vertebral/cirugía; Anestesia; Seguridad; Complicaciones; Posicionamiento del paciente; Posición prona.

\section{INTRODUÇÃO}

As cirurgias da coluna vertebral realizadas em decúbito ventral e com anestesia geral, acarretam riscos para o paciente em decorrência do posicionamento da cabeça durante o ato operatório. São descritos na literatura, isquemia do nervo ótico ${ }^{1}$, amaurose ${ }^{2-7}$, oclusão da artéria vertebral ${ }^{8}$, necrose de pele por pressão ${ }^{8}$ e déficit neurológico secundário a compressão medular ${ }^{8}$, especialmente nos pacientes idosos com espondilose cervical, quando submetidos à rotação lateral da coluna cervical.

Para minimizar os riscos destas complicações, criamos um suporte para colocação da cabeça do paciente em alinhamento neutro com o corpo, que permite ao anestesista adequada avaliação e acompanhamento dos olhos, boca, nariz e do tubo oro-traqueal, durante a cirurgia.

Este suporte foi desenvolvido em 2008, com recursos próprios dos autores, e desde então é utilizado como item padrão nas cirurgias realizadas pela nossa equipe.

\section{DESCRIÇÃO DO SUPORTE}

O suporte é composto de duas peças; um coxim em forma de ferradura e uma base em fibra de polietileno com espelho inclinado removível (Figura 1a,b,c).

O coxim em forma de ferradura é composto por três peças. A espuma, densidade 28, recortada na forma acima citada, com 6 centímetros de altura e $6 \mathrm{~cm}$ de largura. É suportada por peça de polietileno rígido, de $1,5 \mathrm{~cm}$ de espessura, também na forma acima citada.

A ferradura tem $29 \mathrm{~cm}$ de comprimento, diâmetro externo de $18 \mathrm{~cm}$ na base,o diâmetro externo na altura dos olhos com $25 \mathrm{~cm}$ e o diâmetro externo distal de $20 \mathrm{~cm}$. O vão livre tem $23 \mathrm{~cm}$ de comprimento e diâmetro interno máximo de $11 \mathrm{~cm}$, altura dos olhos. A parte distal do coxim possui uma chanfradura que permite ao anestesista a conferencia das estruturas da face. A espuma e o polietileno são revestidos por tecido em napa branca cirúrgica.

Para a conexão com a base em fibra de polietileno, foi desenhada uma peça em aço inox com quatro colunas maciças de $6,5 \mathrm{~cm}$ de comprimento e $0,8 \mathrm{~cm}$ de diâmetro, que deslizam dentro de outras quatro colunas, $6,5 \mathrm{~cm}$ por $1 \mathrm{~cm}$ de diâmetro, (vazada) fixadas na base. Este item de regulação de altura permite ao cirurgião e ou anestesista fletir ou estender a cabeça do paciente, para melhor relaxamento das estruturas cervicais. As distancias entre as coluna posteriores é de $17,5 \mathrm{~cm}$ e entre as anteriores 11 $\mathrm{cm}$. A distancia entre a posterior e anterior ipsilateral é de $7,5 \mathrm{~cm}$, formando um trapézio.

A base em fibra de polietileno, $1,5 \mathrm{~cm}$ de espessura, também é revestida de napa. Apresenta $27 \mathrm{~cm}$ de comprimento por $26 \mathrm{~cm}$ de largura com cantos arredondados. A peça de aço inox com as quatro colunas é deslocada para a frente da base colocada a uma

\footnotetext{
1. Ortopedista, pesquisador do Laboratório de Anatomia Neurocirúrgica da Faculdade de Medicina da Universidade Federal de Minas Gerais - UFMG, Minas Gerais, Brasil.

2. Neurocirurgião, Professor Substituto do Departamento de Cirurgia da Faculdade de Medicina da UFMG - Minas Gerais, Brasil.

3. Anestesista, Professor Assistente da Faculdade de Medicina da Universidade Federal de Minas Gerais - UFMG, Minas Gerais, Brasil.

4. Ortopedista, Chefe do serviço de Cirurgia da Coluna do hospital Madre Teresa de Belo Horizonte, Minas Gerais, Brasil.

5. Ortopedista, Chefe do serviço de Cirurgia da Coluna do hospital Maria Amélia Lins - Fundação Hospital do Estado de Minas Gerais (FHEMIG) - Minas Gerais, Brasil.

6. Neurocirurgião, ProfessorTitular do Departamento de Cirurgia da Faculdade de Medicina da Universidade Federal de Minas Gerais - UFMG, Minas Gerais, Brasil.
}

Trabalho realizado no Laboratório de Anatomia Neurocirúrgica da Faculdade de Medicina da Universidade Federal de Minas Gerais.MG.Brasil.

Correspondência:Faculdade de Medicina - Av. Alfredo Balena, n 190, Sala 127. CEP: 30.130-100. Belo Horizonte. Minas Gerais. Brasil. guizanini@ yahoo.com.br 
distancia de $2,5 \mathrm{~cm}$ da borda distal. Isto permite que a base entre por baixo do dispositivo que sustenta o abdome e tórax (coxins, bóia, ou suporte de espuma), aumentando a estabilidade.

O espelho tem a forma trapezoidal com $15 \mathrm{~cm}$ de altura; a base maior com $18 \mathrm{~cm}$ e a base menor com $14 \mathrm{~cm}$. Ele é colocado em trilho inclinado de $15 \mathrm{~cm}$ de comprimento e 15 graus de inclinação no plano horizontal, removível para facilitar a assepsia do conjunto. A inclinação do espelho permite ao anestesista visualização dos olhos, nariz, tubo endotraqueal e boca, confirmando se os mesmos estão protegidos e livres de compressão durante o tempo cirúrgico (Figura 2).

Outra característica do suporte é que a cabeça não é fixada, permite assim, cirurgias realizadas sobre tração.

Trata-se de material em aço inox, de longa duração, não descartável. É lavável e permite a anti-sepsia por meios químicos, incluindo do espelho destacável.
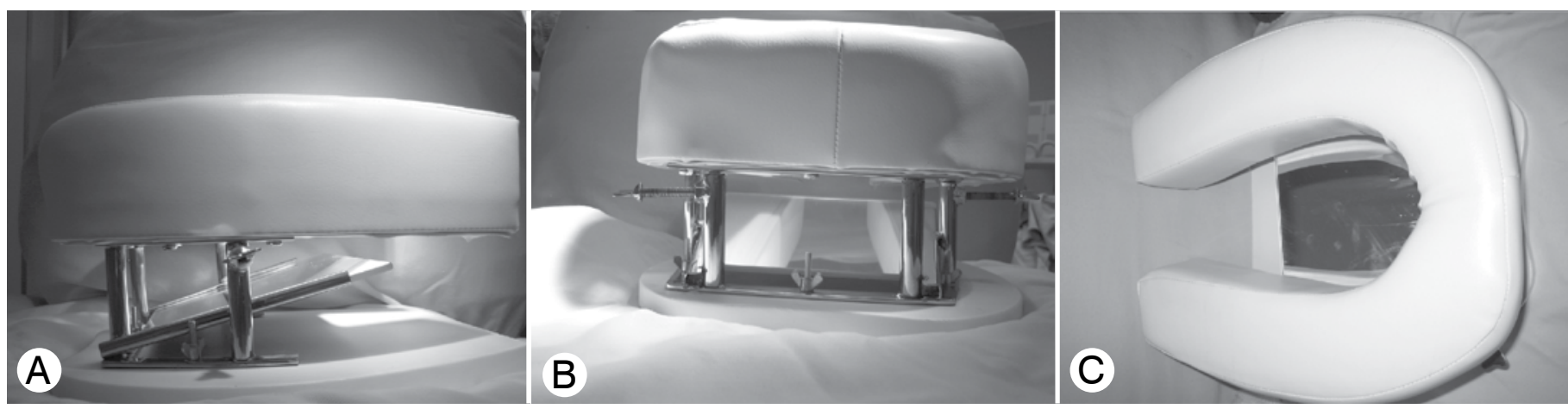

FIGURA 1 - Suporte de cabeça: A) vista lateral, B) vista anterior, C) vista superior.

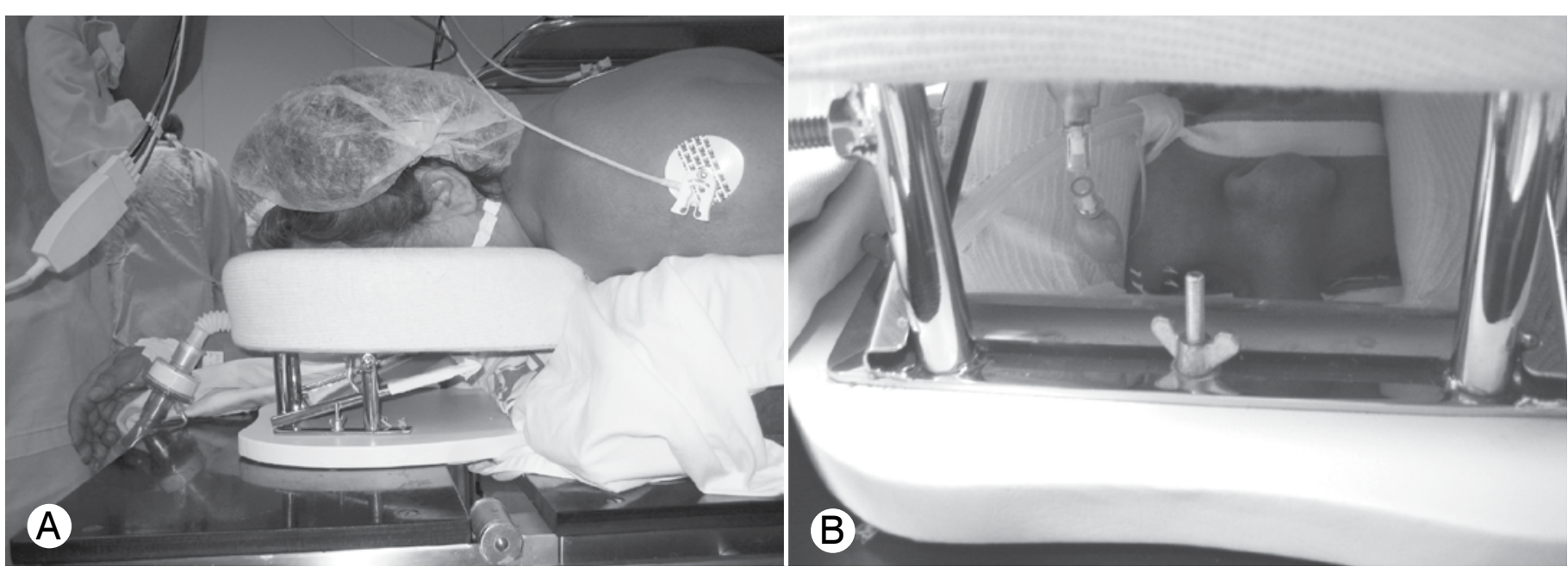

FIGURA 2 - A) Paciente posicionado para cirurgia da coluna torácica, nota-se o alinhamento neutro da cabeça em relação ao eixo do corpo. B) visão de olhos boca e nariz pelo espelho inclinado.

\section{REFERÊNCIAS}

1. Corso CM, Tanaka PP. Isquemia do nervo óptico após intervenção cirúrgica na coluna vertebral: relato de caso. Rev Bras Anestesiol. 2006;56(3): 273-7.

2. Warner ME, Warner MA, Garrity JA, MacKenzie RA, Warner DO. The frequency of perioperative vision loss. Anesth Analg. 2001;93(6):1417-21.

3. Myers MA, Hamilton SR, Bogosian AJ, Smith $\mathrm{CH}$, Wagner TA. Visual loss as a complication of spine surgery. A review of 37 cases. Spine (Phila Pa 1976). 1997:22(12):1325-9.

4. Roth S, Nunez R, Schreider BD. Unexplained visual loss after lumbar spinal fusion. J Neurosurg Anesthesiol. 1997:9(4):346-8.
5. Lee LA, Lam AM. Unilateral blindness after prone lumbar spine surgery. Anesthesiology. 2001;95(3):793-5.

6. Yi HJ, Kim DW. Reversible unilateral blindness after lumbar spine surgery: a role for cerebrospinal fluid leakage? J Neurosurg Anesthesiol. 2004;16(4):322-3.

7. Cheng MA, Todorov A, Tempelhoff R, McHugh T, Crowder CM, Lauryssen C. The effect of prone positioning on intraocular pressure in anesthetized patients. Anesthesiology. 2001:95(6):1351-5.

8. Warner MA. Perioperative neuropathies, blindness and positioning problems. ASA Annual Meeting Refresher Course Lectures. 2004;L117:1-7. 